Einführung zum Thema

Gastroenterologe $2021 \cdot 16: 1-2$

https://doi.org/10.1007/s11377-020-00497-w

Angenommen: 3. Dezember 2020

(c) Springer Medizin Verlag $\mathrm{GmbH}$, ein Teil von Springer Nature 2020

In den letzten Jahrzehnten hat durch die rasante Weiterentwicklung der digitalen Möglichkeiten in vielen Bereichen der Wirtschaft, aber auch in den naturwissenschaftlichen Fächern sog. künstliche Intelligenz (KI) Einzug gehalten. Diese Entwicklungen haben bereits - oft unbemerkt im Hintergrund - schon jetzt Auswirkungen auf das Leben vieler Menschen. In der Industrie werden vernetzte Roboter eingesetzt, die Produkte herstellen und bearbeiten - mit einer Präzision, die von Menschen nicht durchgehend erreicht werden kann. Denken wir an das „autonome Fahren“, das hochkomplexe Zusammenhänge zwischen den Fahreigenschaften des Autos, Erkennung der nahen und fernen Umgebung des Fahrzeugs und den Abgleich mit gesetzlichen Regeln (z. B. Verkehrsordnung) in Echtzeit voraussetzt. Auf der anderen Seite werden vollautomatisch Menschenmassen per Gesichtserkennung überwacht und Individuen mit hoher Sicherheit identifiziert und kontrolliert.

In einem aktuellen Interview in der FAZ (Frankfurter Allgemeine Zeitung) werden Ergebnisse einer repräsentativen Befragung von 1000 jungen Menschen zwischen 16 und 30 Jahren wiedergegeben. Auf die Frage, ob der Einsatz von KI eher als Wunschvorstellung oder als Horrorszenario gilt, „können sich $43 \%$ ein besseres Leben durch KI vorstellen, $42 \%$ standen dem eher neutral gegenüber, $8 \%$ sagten sie wüssten es nicht und nur $7 \%$ halten es für ein Horrorszenario". Bei den mittelalten und älteren Menschen ist die Ambivalenz wahrscheinlich deutlich größer [1].

\author{
R. Jakobs' $\cdot$ M. Fried ${ }^{2} \cdot$ J. Hampe ${ }^{3}$ \\ ${ }^{1}$ Medizinische Klinik C, Klinikum der Stadt Ludwigshafen, Ludwigshafen, Deutschland \\ ${ }^{2}$ Abteilung Gastroenterologie und Hepatologie, Universitätsspital Zürich, Zürich, Schweiz \\ ${ }^{3}$ Medizinische Klinik1, Universitätsklinikum Dresden, Dresden, Deutschland
}

\title{
Künstliche Intelligenz in der Viszeralmedizin - „brave new world" oder digitaler Horror?
}

Für die Medizin ergibt sich ein ähnliches Spannungsfeld. Durch neuronale Netzwerke werden zukünftig neue Applikationen in der Medizin möglich und wahrscheinlich die traditionelle Vorstellung von Medizin disruptiv verändern. Es sind bereits Smartphone-Apps im Einsatz, die mit großer Genauigkeit in der Lage sind, einen Hautnaevus zu analysieren und die Dignität einzuschätzen. Netzhautscans erlauben die automatisierte Erkennung von retinalen Erkrankungen, aber auch sekundärer Erkrankungen wie Hypertonie oder Diabetes.

In dieser Ausgabe von Der Gastroenterologe werden in 3 Beiträgen Aspekte der Anwendung künstlicher Intelligenz in der Viszeralmedizin vorgestellt.

Für die Endoskopie existieren bereits Anwendungen, die eine Erkennung von malignen Läsionen im oberen Gastrointestinaltrakt ermöglichen [2]. Die automatisierte Erfassung der Untersuchungszeit durch Detektion anatomischer Landmarken und die Auswertung der tatsächlich im Bild erfassten Anteile der Mukosa sind schon experimentell möglich [3,4]. Die aktuellen Entwicklungen der KI in der Vorsorgekoloskopie und Krebsfrüherkennung am Dickdarm fassen HansDieter Allescher et al. zusammen. Neben der reinen Detektion von Kolonpolypen wird durch die neuen technischen Entwicklungen die direkte Klassifikation der erkannten Läsionen und somit die Abschätzung der Dignität ermöglicht. Die klinischen Auswirkungen dieser neuen Optionen auf die bereits hervorragenden Effekte der Vorsorgekoloskopie bleiben abzuwarten.
Die Sedierung von Patienten in der Endoskopie erfolgt heute standardmäBig mit Propofol durch speziell geschulte Pflegekräfte („,nurse-administered“) oder intensivmedizinisch erfahrene Ärzte. Ideal wäre ein geschlossenes System, das automatisch über valide Messparameter die Propofolapplikation und damit die Sedierungstiefe steuert. Die Möglichkeiten und Limitationen einer automatisierten KI-basierten Sedierungssteuerung über Elektroenzephalographie (EEG) wird von J. Garbe und V. Garbe et al. vorgestellt.

Die OP-Robotik hat in den letzten 10 Jahren bereits die Prostataresektion revolutioniert, auch in der Viszeralmedizin scheinen Vorteile bei der Operation im kleinen Becken (z. B. Resektion tiefsitzender Rektumkarzinome), aber auch bei der Ösophagusresektion zu bestehen. Bereits jetzt können viele individuelle Daten zu operativen Strategien beim Einsatz der Robotikgesammelt werden. Zumindest theoretisch könnte über die Auswertung vieler Eingriffe und unterschiedlicher Operateure weltweit ein großer eingriffsspezifischer Erfahrungsschatz aufgebaut werden, der einem OPRoboter die autonome Operation erlauben könnte.

Wollen Sie von diesem super-erfahrenen Kollegen OP-Roboter autonom operiert werden? Wie Franziska Mathis-Ullrich, ausgewiesene Expertin in diesem Bereich, die Situation der OP-Robotik aktuell und in naher Zukunft sieht, wird nicht nur den Gastroenterologen, sondern wahrscheinlich auch den Viszeralchirurgen interessieren. 
Bei der Vielzahl und Rasanz der neuen technischen Entwicklungen werden wahrscheinlich in Kürze weitere Anwendungen zur Marktreife gebracht, die neben der Endoskopie auch die Diagnostik bei Lebererkrankungen, Labordiagnostik, Bildgebung und Pathologie erheblich verändern und vielleicht auch in Teilbereichen verbessern dürften. Damit ergeben sich aber auch immer mehr ethische Fragen und Aspekte, unter anderem ob und wie autonom KI-Systeme Menschen behandeln können (und sollten) und ob ein Mediziner „nur mit Menschenverstand“ (und ohne KI) zukünftig noch rechtssicher behandeln kann und darf.

Die Diskussion und möglicherweise Einigung auf Regeln zu ethischen Aspekten dieser neuen Applikationen setzen einen durch gute Informationen gestützten Diskurs bei den potenziellen Anwendern und der Gesellschaft voraus. Diese Ausgabe von Der Gastroenterologe soll zumindest Teilbereiche der Anwendung künstlicher Intelligenz in der Viszeralmedizin vorstellen.

Wir wünschen Ihnen viel Freude und Erkenntnisgewinn bei der Lektüre!

\section{Korrespondenzadresse}

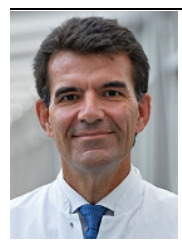

Prof. Dr. R. Jakobs

Medizinische Klinik

C, Klinikum der Stadt

Ludwigshafen

Bremserstraße 79,

67063 Ludwigshafen,

Deutschland

jakobsr@klilu.de

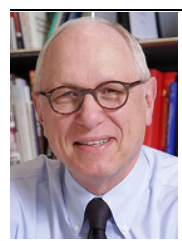

Prof. Dr. M. Fried

Abteilung Gastroenterologie und Hepatologie,

Universitätsspital Zürich

Rämistr. 100, 8091 Zürich,

Schweiz

michael.fried@usz.ch

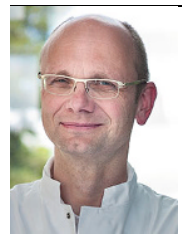

Prof. Dr. J. Hampe

Medizinische Klinik1, Universitätsklinikum Dresden Fetscherstraße 74, 01307 Dresden, Deutschland jochen.hampe@ uniklinikum-dresden.de

Interessenkonflikt. R. Jakobs, M. Fried und J. Hampe geben an, dass kein Interessenkonflikt besteht.

\section{Literatur}

1. Preuß M, Bös N (2020) Mehr Zeit für Kreativität! Frankf Allg Ztg 278:C2

2. Cai SL, Li B, Tan WM, Niu XJ et al (2019) Using deep learning system in endoscopy for screening of early esophageal squamous cell carcinoma (with video). GastrointestEndosc90(5):745-753.e2. https://doi. org/10.1016/j.gie.2019.06.044

3. Wu L, Zhang J, Zhou W, An P, Shen L, Liu Jetal (2019) Randomised controlled trial of WISENSE, a realtime quality improving system for monitoring blind spots during esophagogastroduodenoscopy. Gut 68:2161-2169. https://doi.org/10.1136/ gutjnl-2018-317366

4. Abadir AP, Ali MF, Karnes W, Samarasena JB (2020) Artificial intelligence in gastrointestinal endoscopy. Clin Endosc 53:132-141. https://doi. org/10.5946/ce.2020.038

\section{Gastro Update Livestream: Innovativ und flexibel}

12. und 13. März aus Berlin 19. und 20. März aus Mainz

Umfangreiches Update-Wissen in schwierigen Zeiten: Erstmals können sich interessierte Ärzte für das komplette Gastro-Update-Seminar zum innovativen Livestream anmelden. Seien Sie LIVE dabei, bequem von zu Hause oder aus der Klinik! Die Interaktivität wird durch einen Livechat ermöglicht und die Zertifizierung ist beantragt, sodass Sie auch bei virtueller Teilnahme Ihre wichtigen CME-Punkte erhalten.

Das Update-Konzept: Die wichtigsten Neuerungen des vergangenen Jahres aus der Gastroenterologie werden unter der wissenschaftlichen Leitung von Prof. Dr. Peter Layer (Hamburg), Prof. Dr. Thomas Berg (Leipzig), Prof. Dr. Andrea May (Wiesbaden) und Prof. Dr. Andreas Stallmach (Jena) kritisch selektiert, analysiert und zusammengefasst. Die Relevanz für den Klinik- und Praxisalltag der Ärzte steht dabei im Vordergrund und der ausführlichen Diskussion über den Livechat mit den Referenten wird viel Raum gegeben.

In diesem Jahr werden neben den Kerngebieten der Gastroenterologie die Hot Topics »Psychosomatik« und »Fehlernährung" vorgestellt.

Zum Gesamtpaket der Teilnahme gehören neben den umfangreichen Seminarunterlagen als eBook der Download aller Vortragspräsentationen und die Vorträge im Nachgang als Video-on-Demand auf der Videoplattform »streamed-up.com«.

Weitere Informationen zu Programm, Referenten und zur Anmeldung:

www.gastro-update.com

Veranstalter: med update $\mathrm{GmbH}$ Hagenauer Straße 53 65203 Wiesbaden 